\title{
DTI studies in patients with Alzheimer's disease, mild cognitive impairment, or normal cognition with evaluation of the intrinsic background gradients
}

\author{
Geon-Ho Jahng • Songfan Xu • Micheal W. Weiner • \\ Dieter J. Meyerhoff • Seungjoon Park • Norbert Schuff
}

Received: 25 September 2010 / Accepted: 1 February 2011 / Published online: 22 February 2011

(C) The Author(s) 2011. This article is published with open access at Springerlink.com

\begin{abstract}
Introduction The objective of the study was to explore the impact of the background gradients on diffusion tensor (DT) magnetic resonance imaging (DT-MRI) in patients with Alzheimer's disease (AD), mild cognitive impairment (MCI), or cognitively normal (CN) aging.

Methods Two DT-MRI sets with positive and negative polarities of the diffusion-sensitizing gradients were obtained in $15 \mathrm{AD}$ patients, $18 \mathrm{MCI}$ patients, and $16 \mathrm{CN}$ control subjects. The maps of mean diffusivity (MD) and fractional anisotropy (FA) were computed separately for
\end{abstract}

G.-H. Jahng ( $\bowtie)$

Department of Radiology,

Kyung Hee University Hospital-Gangdong,

School of Medicine, Kyung Hee University,

149 Sangil-dong, Gangdong-gu,

Seoul 134-727, South Korea

e-mail: ghjahng@gmail.com

S. Xu $\cdot$ S. Park

Department of Biomedical Science,

Graduate School of Kyung Hee University,

1 Hoegi-dong, Dongdaemun-gu,

Seoul 130-701, South Korea

M. W. Weiner · D. J. Meyerhoff $\cdot$ N. Schuff

Center for Imaging of Neurodegenerative Diseases,

VA Medical Center and the Department of Radiology,

University of California-San Francisco,

4150 Clement Street, 114M,

San Francisco, CA 94121, USA

\section{S. Park}

Department of Pharmacology and the Medical Research Center for Bioreaction to ROS and the Biomedical Science Institute,

School of Medicine, Kyung Hee University,

1 Hoegi-dong, Dongdaemun-gu,

Seoul 130-701, South Korea positive (p: pMD and pFA) and negative (n: $\mathrm{nMD}$ and $\mathrm{nFA}$ ) polarities, and we computed the geometric mean (gm) of the DT-MRI to obtain the gmFA and gmMD with reducing the background gradient effects. Regional variations were assessed across the groups using one-way analysis of variance.

Results Increased regional gmMD values in the $\mathrm{AD}$ subjects, as compared to the regional gmMD values in the MCI and CN subjects, were found primarily in the frontal, limbic, and temporal lobe regions. We also found increased $\mathrm{nMD}$ and $\mathrm{pMD}$ values in the $\mathrm{AD}$ subjects compared to those values in the MCI and $\mathrm{CN}$ subjects, including in the temporal lobe and the left limbic parahippocampal gyrus white matter. Results of comparisons among the three methods showed that the left limbic parahippocampal gyrus and right temporal gyrus were the increased $\mathrm{MD}$ in the $\mathrm{AD}$ patients for all three methods.

Conclusion Background gradients affect the DT-MRI measurements in AD patients. Geometric average diffusion measures can be useful to minimize the intrinsic local magnetic susceptibility variations in brain tissue.

Keywords Diffusion tensor imaging · Background gradients · Alzheimer's disease $\cdot$ Mild cognitive impairment $\cdot$ Geometric mean analysis

\section{Introduction}

Diffusion tensor (DT) magnetic resonance imaging (DTMRI) is sensitive to the directionality of the random motion of water in tissue, and it involves the application of external diffusion-sensitizing magnetic field gradients along different orientations to quantify the properties of diffusion. 
Numerous DT-MRI studies of neurodegenerative diseases have reported abnormal diffusion values in the brain, including Alzheimer's disease (AD), which is a devastating condition that leads to progressive memory loss and rapid cognitive decline. Although $\mathrm{AD}$ is generally considered to affect primarily the gray matter, several studies have found changes of the isotropic and anisotropic diffusion in white matter associated with AD progression by using DT-MRI [1-6]. The diffusion abnormalities in $\mathrm{AD}$ were predominantly found in the posterior regions of the brain such as the hippocampal gyrus, the temporal white matter, the splenium of the corpus callosum, and the posterior cingulum. In patients with mild cognitive impairment (MCI), which is considered to represent a transitional stage between normal aging and $\mathrm{AD}$, the changes seem to parallel those in $\mathrm{AD}$ with similar posterior regions showing abnormalities. In contrast to $\mathrm{AD}$ and $\mathrm{MCI}$, the diffusion abnormalities in subjects with age-associated changes (cognitively normal, $\mathrm{CN}$ ) occur in the frontal regions, and specifically in the frontal white matter, the anterior cingulum, and the genu of the corpus callosum [7].

Although the marked differences seen on DT-MRI between $\mathrm{AD}$ or MCI and normal aging have been considered as potential imaging markers [8-10], the underlying mechanism of the DT-MRI changes remains largely unexplained. In particular, the local variations in cell density, oligodentrocytes, myelination, and also amyloid plaques, which are a hallmark of $\mathrm{AD}$ [11-13], can be the source of local magnetic susceptibility variations, which in turn can alter water diffusion. Furthermore, it has been shown in rat brain [14] that brain iron, which occurs in high concentrations in oligodentrocytes and plaques [15-17], can modulate the diffusion measurements. These finding suggests that local magnetic susceptibility variations in brain tissue may contribute to the DT-MRI abnormalities seen in $\mathrm{AD}$ and MCI in the form of intrinsic susceptibilitydependent background gradients that add to the external diffusion weighting gradients. The previous DT-MRI studies did not take into account the local variations in brain.

All investigators may be interested in knowing where in the brain and for which patients these different diffusion effects are with and without considering the background gradients. So, the overall goal of this study was to investigate whether intrinsic background gradients contribute to the pattern of regional diffusion abnormalities in patients with $\mathrm{AD}, \mathrm{MCI}$, and $\mathrm{CN}$, and this potentially reflects the underlying pathological processes associated with brain iron. Specifically, we hypothesized that $\mathrm{AD}$ patients show a systematic pattern of higher regional background gradients compared to the $\mathrm{MCI}$ and $\mathrm{CN}$ subjects.

\section{Materials and methods}

\section{Theoretical background}

Neeman et al. [18] reported the use of diffusion-encoding schemes that were made up of couples of gradients with positive and negative polarities in order to minimize a cross-term effect in the case of static field inhomogeneities. To minimize the scalar effects of the unknown cross-term effect of the background and the diffusion-encoding gradients, the geometric mean (gm) operation was used for both the positive and negative polarities of the diffusion-encoding gradients by applying the following equations $[18,19]$ :

$S_{g m}=\sqrt{S_{p} \cdot S_{n}}=S_{0} \operatorname{Exp}\left(-b_{g m} \cdot A D C\right)$

$b_{g m}=\frac{b_{p}+b_{n}}{2}=-\gamma^{2} \delta^{2}\left(a G_{d}^{2}+b G_{b}^{2}+c G_{\text {img }}^{2}+f G_{b} \cdot G_{i m g}\right)$

$b_{g m} \cong-\gamma^{2} \delta^{2}\left(a G_{d}^{2}+c G_{i m g}^{2}\right)$

where the geometric mean operation is defined as $\sqrt{S_{p}{ }^{*} S_{n}}$ where $S_{\mathrm{p}}$ and $S_{\mathrm{n}}$ are the signals acquired with positive (p) and negative (n) gradients polarities, $S_{0}$ is the signal acquired without a diffusion gradient, the $b$ value $=0 \mathrm{~s} /$ $\mathrm{mm}^{2}, b_{\mathrm{p}}$ is the $b$ value with using positive diffusion gradients, $b_{\mathrm{n}}$ is the $b$ value with using negative diffusion gradients, $b_{\mathrm{gm}}$ is the $b$ value calculated by $b_{\mathrm{p}}$ and $b_{\mathrm{n}}, \gamma$ is the gyromagnetic ratio, $\delta$ is the duration of the externally applied diffusion gradient, $G_{\mathrm{d}}$ is the amplitude of the known diffusion-encoding gradients, $G_{\mathrm{b}}$ is an amplitude of the unknown background gradients, $G_{\text {img }}$ is the amplitude of the known imaging gradients, and $a, b, c$, and $f$ are coefficients. Please note that with this calculation, the cross terms, both $G_{\mathrm{b}} * G_{\mathrm{d}}$ and $G_{\mathrm{img}} * G_{\mathrm{d}}$, disappeared. The background gradients $G_{b}^{2}$ and the cross term, $G_{\mathrm{b}} * G_{\text {img }}$, in Eq. 2 can be ignored because those values are much smaller than $G_{d}^{2}$. Therefore, the $b_{\mathrm{gm}}$ value can finally be considered as Eq. 3.

\section{Subjects}

Table 1 lists the demographic data of the subjects, including gender, age, and the Mini-Mental State Examination Score (MMSE), which is a general measure of cognitive performance. Fifteen patients diagnosed with $\mathrm{AD}$ (mean age 75 years, standard deviation (SD) 9.2, age range 6186 years, 9 males and 6 females, MMSE range 7-28, mean MMSE 21.9) based on the National Institute of Neurolog- 
Table 1 Demographic data and the neuropsychologic test results

\begin{tabular}{llll}
\hline & AD & MCI & CN \\
\hline Subjects & 15 & 18 & 16 \\
Age $^{\mathrm{a}}$ (years) & $76.6(9.1)$ & $72.6(8.5)$ & $73.1(9.5)$ \\
Gender & & & \\
Male & 9 & 7 & 9 \\
Female & 6 & 11 & 7 \\
MMSE & $21.9(5.5)^{\mathrm{b}}$ & $28.5(1.8)$ & $29.3(0.8)$ \\
\hline
\end{tabular}

$A D$ Alzheimer's disease, $M C I$ mild cognitive impairment, $C N$ cognitively normal, MMSE Mini-Mental State Examination Score

The data are presented as the mean (standard deviation)

${ }^{a}$ There are no statistically significant differences between the groups $(p>0.05)$

${ }^{\mathrm{b}}$ There are statistically significant differences between the AD group and the other groups $(p<0.0005)$, but not between the MCI and CN groups $(p>0.215)$

ical and Communicative Disorders and Stroke-Alzheimer's Disease and Related Disorder's Association (NINCDSADRDA) criteria were studied using a $1.5 \mathrm{~T}$ clinical MRI system. In addition, 18 patients diagnosed with MCI (mean age 72 years, SD 8.5, age range 56-96 years, 7 males and 11 females, MMSE range 23-30, mean MMSE 28.5) and $16 \mathrm{CN}$ control subjects (mean age 73 years, SD 9.5, age range 62-85 years, 9 males and 7 females, MMSE range 28-30, mean MMSE 29.3) were recruited as well. The diagnosis of MCI followed Petersen's criteria [20]. Prior to the onset of this study, informed consent was obtained from all subjects, and the protocol was approved by the local institutional review board in the USA. All experiments on human subjects were conducted in accordance with the Declaration of Helsinki.

\section{MRI acquisition}

The DT-MRI measurements were performed using a singleshot echo-planar imaging (EPI) sequence with inversionprepared magnetization to suppress the cerebrospinal fluid (CSF) [21]. CSF suppression was used to reduce errors in the diffusion measurements from the partial volume effects in the voxels that represent CSF. A double refocusing spinecho acquisition with bipolar external diffusion gradients [22] was employed to minimize the artifacts due to eddy currents. Six diffusion encoding directions [23] and five diffusion sensitivities ( $b$ values $0,160,360,640$, and $1,000 \mathrm{~s} / \mathrm{mm}^{2}$ ) were acquired to determine the apparent diffusion coefficients and the diffusion tensor for each voxel. Furthermore, two DT-MRI datasets were acquired with alternating polarities of the external diffusionsensitizing gradients (positive $+G_{\mathrm{d}}$ and negative $-\mathrm{G}_{\mathrm{d}}$ ) to investigate the effects of background gradients on the DT-
MRI measures of the patients with AD. The other imaging parameters were as follows: repetition time (TR)/echo time (TE)/inversion time $(\mathrm{TI})=5,000 / 100 / 3,000 \mathrm{~ms}$ with $2.4 \times$ $2.4 \mathrm{~mm}^{2}$ in-plane resolution and 19 slices of 5-mm slice thickness without a gap, which covered approximately $80 \%$ of the brain.

In addition to the DT-MRI scan, the sagittal structural volumetric $\mathrm{T}_{1}$-weighted (T1W) images were acquired as follows: $\mathrm{TR} / \mathrm{TE} / \mathrm{TI}=10 / 4 / 300 \mathrm{~ms}$, flip angle $=15^{\circ}$ and spatialresolution $=1 \times 1 \times 1.5 \mathrm{~mm}$ resolution. The intermediate (or proton density)-weighted (PD) and $\mathrm{T}_{2}$-weighted (T2W) axial images were also acquired using a multislice double spin-echo (DSE) sequence. The imaging parameters for DSE were as follows: TR/TE $/=5,000 / 20 / 80 \mathrm{~ms}$ with $1.25 \times 1 \mathrm{~mm}$ in-plane resolution and a $3-\mathrm{mm}$ thickness, and contiguous slices covering the entire brain. The structural images allowed registration between the structural data and the DT-MRI data and spatial normalization of the DT-MRI indices into a reference space (vide infra). In addition, a $\mathrm{T}_{2^{-}}$ weighted spin-echo EPI image (referred to below as a reference EPI image) was acquired at the same resolution and orientation as the diffusion scans, but with whole brain coverage and without inversion preparation to improve registering the DT-MRI data to the structural images. Acquisition of the reference EPI was necessary because the DT-MRI slices did not cover the whole brain and the EPI data without diffusion gradients (EPI at $b=0 \mathrm{~s} / \mathrm{mm}^{2}$ ) had limited structural information because of the inversion pulse.

\section{DT-MRI preprocessing}

In order to map the diffusion indices of the mean diffusivity (MD) and the fractional anisotropy (FA) from the DT-MRI data obtained from the positive $\left(+G_{\mathrm{d}}\right)$ and negative $\left(-G_{\mathrm{d}}\right)$ diffusion-encoding gradients, we developed in house software with interactive data language (IDL; Research Systems, Inc., Boulder, CO). The MD and FA maps (pMD and pFA) were calculated by using only the positive polarity of the diffusion-encoding gradients, and these maps (nMD and nFA) were also calculated by using only the negative polarity of the diffusion-encoding gradients separately without taking into account a background gradient effect. In addition, the maps of the geometric means of MD and FA (gmMD and gmFA) were calculated to minimize the unknown cross-term effects of the background and the diffusion-encoding gradients. The geometric means of FA and MD were calculated by taking the inner product of the diffusion-encoding gradients with positive and negative polarities according to $\sqrt{S_{p} * S_{n}}$, where $S_{\mathrm{p}}$ and $S_{\mathrm{n}}$ are the diffusion-weighted signals acquired with the positive (p) and negative (n) gradient polarities, respectively [18, 19]. The calculations of the geometric 
mean used the same $b$ values as the ones used for the DTMRI with the positive and negative diffusion-encoding gradients. A more detailed description of the geometrical mean computation can be found in the "Theoretical background" section. All the maps were calculated before performing imaging coregistration.

Postprocessing and statistical analyses

\section{Image coregistration}

The raw DT-MRI and DT_b0 EPI were assumed to be intrinsically aligned. This assumption is reasonable because first, both datasets are subjected to similar geometrical distortions; second, additional distortions due to the eddy currents induced by the diffusion-encoding gradients are minimal since we used a double refocusing sequence; and third, the datasets were acquired in an interleaved fashion to reduce movement effects between the frames. The DT_b0 EPI data $(\mathrm{TE}=100 \mathrm{~ms}$ and inversion-prepared and 19 slices) were coregistered to the reference EPI data (TE= $100 \mathrm{~ms}$ without inversion, but with whole brain coverage) using an affine transformation available with Statistical Parameter Mapping software (SPM2, Wellcome Department of Cognitive Neurology, England, UK). The reference EPI data were then further coregistered to the $\mathrm{T} 2 \mathrm{~W}$ images, which were in turn coregistered to the PD images (acquired together with the $\mathrm{T} 2 \mathrm{~W}$ using double spin-echo acquisition). Finally, the PD images, and thus the DT_b0 EPI data and the maps of the DT-MRI data were coregistered to the 3D $\mathrm{T} 1 \mathrm{~W}$ images. These steps allowed a reliable coregistration between the MD and FA maps and the anatomical 3D T1W images.

\section{Spatial normalization}

A study-specific template was created by transforming the 3D T1W images from all the subjects in this study into a T1W SPM2 template space using affine transformations and by averaging all the transferred 3D T1W images provided from the SPM2 website. We created this template since our study population had brain disease and the study population was considerably older than the populations used in the standard templates, such as the Montréal Neurological Institute (MNI) template. After creating the study specific template, all the $3 \mathrm{D} \mathrm{T} 1 \mathrm{~W}$ images from the individual subjects were again spatially normalized to this study specific template using a 12-parameter nonlinear transformation $[24,25]$. The same transformation parameters were then applied to normalize all the MD and FA maps, which were also interpolated to the $2 \mathrm{~mm} \times 2 \mathrm{~mm} \times 2 \mathrm{~mm}$ voxel size of the brain template. The maps from the positive and negative external diffusion-sensitizing gradients $(\mathrm{p} / \mathrm{nFA}$ and $\mathrm{p} / \mathrm{nMD}$ ) and the corresponding maps of the geometric means (gmFA and gmMD) were then smoothed using an $8 \times$ $8 \times 12 \mathrm{~mm}$ Gaussian kernel.

\section{Statistical analyses}

In order to investigate the differences in diffusion abnormalities across the groups, voxel-wise one-way analysis of variance (ANOVA) tests were performed on the gmMD and gmFA maps within the framework of SPM2. These analyses were repeated for the pMD and pFA maps as well as for the nMD and nFA maps. In this analysis, we want to know where in the brain and for which patients were the gmMDs different from either the nMD or pMD. To account for multiple comparisons in the voxel-by-voxel tests, the concept of a false discovery rate (FDR) [26] was used, and a threshold for the significance of FDR $p=0.01$ was applied. Based on the results of the tests, we obtained common areas of the three MDs that were $\mathrm{nMD}, \mathrm{pMD}$, and gmMD to evaluate the advantages of the geometrical mean operation.

In addition to the voxel-based analyses, we also analyzed the data with defining a region-of-interest (ROI). The ROIs were defined according to the results of voxel-based analyses. For the MD, the ROIs were the right and left superior temporal gyrus (ROI1), the right and left limbic parahippocampal gyrus (ROI2), and the right and left middle temporal gyrus (ROI3) and the right and left occipital cuneus (ROI4) and the right left limbic uncus (ROI5). For the FA, the ROIs were the right and left superior temporal gyrus (ROI 6,7, and 8) and the right and left superior frontal gyrus (ROI 9, 10). The significant level was used with $p=0.016$ ( $p=0.05 / 3$ times repeated) because we repeated the same tasks three times among the three groups.

\section{Results}

The demographic characteristics of the subjects are summarized in Table 1. There were no significant differences in age and gender across the groups. As expected, the MMSE scores were significantly lower for the $\mathrm{AD}$ patients as compared to those of the other groups $(p<0.05)$, but the MMSE scores did not significantly differ between the MCI and $\mathrm{CN}$ subjects $(p>0.05)$.

Figure 1 shows the results of the voxel-wise comparisons of the $\mathrm{pMD}$, $\mathrm{nMD}$, and gmMD maps between the $\mathrm{AD}$ and $\mathrm{MCI}$ groups (Fig. 1a) and between the $\mathrm{AD}$ and $\mathrm{CN}$ groups (Fig. 1b) based on one-way ANOVA tests. Compared to the MCI patients, the AD patients had increased pMD values mainly in the temporal and frontal lobes. The $\mathrm{AD}$ patients also had increased $\mathrm{nMD}$ values $(\mathrm{AD}>\mathrm{MCI})$ 
Fig. 1 Results of the voxel-wise comparisons of mean diffusivity (MD; $g m M D, n M D, p M D$ ) between the Alzheimer's disease $(A D)$ and mild cognitive impairment $(M C I)$ groups (a) and between the $\mathrm{AD}$ and cognitive normal $(C N)$ groups (b) using one-way ANOVA tests. There were no decreased MD values for all three maps of gmMD, pMD, and $\mathrm{nMD}$ in the patients with $\mathrm{AD}$ as compared with that of the MCI or $\mathrm{CN}$ patients. There were no significant differences between the $\mathrm{MCI}$ and $\mathrm{CN}$ patients a

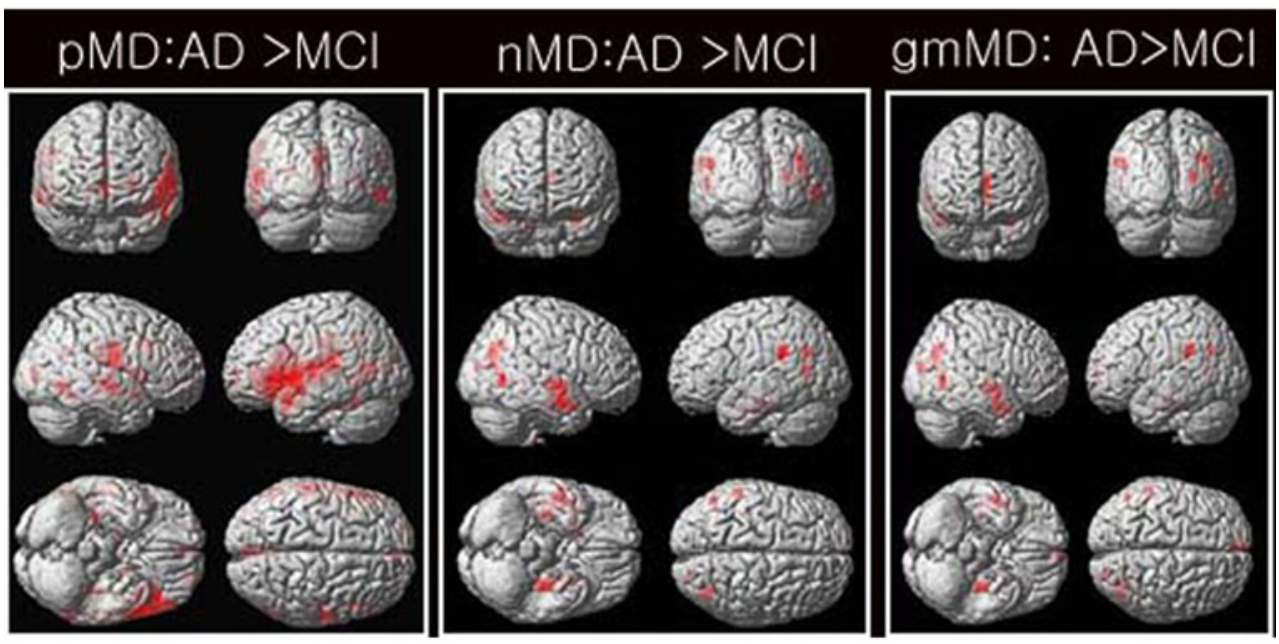

b

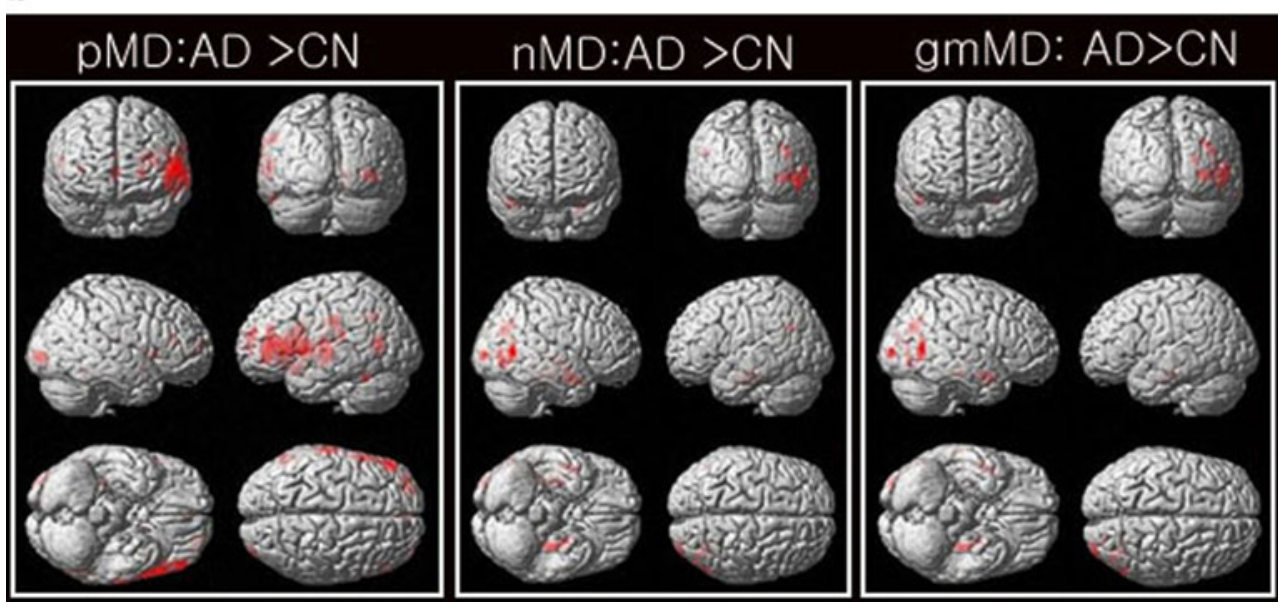

mainly in the temporal lobe. Moreover, we found that the AD patients had increased gmMD values $(\mathrm{AD}>\mathrm{MCI})$ predominantly in the right superior temporal gyrus, the left limbic parahippocampal gyrus white matter, and the left superior and medial frontal gyrus. There were no significantly decreased MD values in the gmMD, pMD, and nMD maps from the patients with $\mathrm{AD}$ as compared with that of the MCI subjects. The detailed results are summarized in Table 2.

Compared to the $\mathrm{CN}$ subjects, the $\mathrm{AD}$ patients had increased pMD values mainly in the temporal gyrus. The $\mathrm{AD}$ patients also had increased nMD values $(\mathrm{AD}>\mathrm{CN})$ mainly in the temporal gyrus. Moreover, we found that the $\mathrm{AD}$ patients had increased gmMD values $(\mathrm{AD}>\mathrm{CN}$, Fig. 1b) predominantly in the left limbic parahippocampal gyrus, the left limbic uncus, the left and right temporal subgyrus, and the right middle temporal gyrus. These detailed results are summarized in Table 3.

Figure 2 shows the differences in the pFA maps between the $\mathrm{MCI}$ or $\mathrm{CN}$ and $\mathrm{AD}$ subjects. Compared to the MCI patients, the $\mathrm{AD}$ patients had increased pFA values $(\mathrm{AD}>$ MCI) mainly in the temporal and frontal gyrus and the posterior cingulate. The detailed results are also summarized in Table 4. Compared to the $\mathrm{CN}$ subjects, the $\mathrm{AD}$ patients also had increased pFA values $(\mathrm{AD}>\mathrm{CN})$ mainly in the left inferior and superior frontal gyrus. We did not find any significant differences in the pFA between the MCI and CN subjects. Similarly, we did not find any significant differences in the gmFA or nFA across the three groups. The results of the FA are summarized in Table 5.

The significant regions that overlap in all three DT-MRI sets (positive gradients, negative gradients, and geometric mean) as well as those regions that differ are listed in Table 6 for the MD (pMD, nMD, and gmMD) and in Table 7 for the FA measures. The overlapping regions of increased $\mathrm{MD}$ in the $\mathrm{AD}$ patients relative to the $\mathrm{MCI}$ subjects $(\mathrm{AD}>\mathrm{MCI})$ included the right superior temporal gyrus and left limbic parahippocampal gyrus. In contrast, the nonoverlapping regions of increased $\mathrm{MD}$ in the $\mathrm{AD}$ patients relative to the $\mathrm{MCI}$ patients $(\mathrm{AD}>\mathrm{MCI})$ included: 
Table 2 The significantly different regions when comparing between the $\mathrm{AD}$ and $\mathrm{MCI}$ groups ( $\mathrm{AD}>\mathrm{MCI}$ ) using the $\mathrm{MD}$ with (gmMD) and without (nMD and $\mathrm{pMD}$ ) minimizing the effects of the background gradients (corrected FDR $(p<0.01)$ as the cluster level; one-way ANOVA tests)

\begin{tabular}{|c|c|c|c|c|c|c|c|c|}
\hline & \multicolumn{3}{|c|}{ Talairach coordinate } & \multirow[t]{2}{*}{ Cluster } & \multirow[t]{2}{*}{$\mathrm{T}$} & \multirow[t]{2}{*}{$\mathrm{Z}$} & \multirow[t]{2}{*}{ Region } & \multirow[t]{2}{*}{ BA ROI\# } \\
\hline & $X$ & $Y$ & $Z$ & & & & & \\
\hline \multirow[t]{8}{*}{ gm } & 50 & -4 & -22 & 602 & 5.56 & 5.27 & R. temporal subgyrus, WM & \\
\hline & 50 & -18 & -4 & 602 & 4.83 & 4.64 & R. superior temporal gyrus, GM & 22 \\
\hline & 58 & -6 & -4 & 602 & 4.8 & 4.61 & R. superior temporal gyrus, WM & ROI1 \\
\hline & -32 & -24 & -22 & 264 & 5.32 & 5.07 & L. limbic parahippocampal gyrus, WM & ROI2 \\
\hline & 38 & -72 & 32 & 323 & 4.94 & 4.73 & R. occipital subgyrus, WM & \\
\hline & -4 & 62 & 14 & 303 & 4.81 & 4.62 & L. superior frontal gyrus, GM & 9 \\
\hline & -4 & 50 & 20 & 303 & 4.65 & 4.47 & L. medial frontal gyrus, WM & \\
\hline & -6 & 62 & -4 & 303 & 4.41 & 4.26 & L. medial frontal gyrus, WM & \\
\hline \multirow[t]{8}{*}{ Neg } & 50 & -6 & -20 & 1,041 & 5.78 & 5.46 & R. temporal subgyrus, WM & \\
\hline & 50 & -18 & -4 & 1,041 & 5.02 & 4.8 & R. superior temporal gyrus, GM & 22 \\
\hline & 58 & -6 & -4 & 1,041 & 5.03 & 4.81 & R. superior temporal gyrus, WM & ROI1 \\
\hline & -30 & -22 & -22 & 546 & 5.73 & 5.41 & L. limbic parahippocampal gyrus, WM & \\
\hline & 38 & -72 & 30 & 432 & 5.18 & 4.95 & R. temporal subgyrus, WM & \\
\hline & 36 & -72 & 6 & 432 & 3.94 & 3.83 & R. middle occipital gyrus, WM & \\
\hline & -26 & -6 & -32 & 546 & 4.35 & 4.21 & L. limbic parahippocampal gyrus, WM & \\
\hline & -32 & 2 & -22 & 546 & 4.49 & 4.33 & L. limbic parahippocampal gyrus, WM & \\
\hline \multirow[t]{18}{*}{ Pos } & 40 & -74 & 30 & 1,759 & 4.82 & 4.63 & R. middle temporal gyrus, WM & ROI3 \\
\hline & -4 & 62 & 10 & 1,024 & 5.38 & 5.11 & L. medial frontal gyrus, GM & \\
\hline & -4 & 50 & 20 & 1,024 & 5.44 & 5.17 & L. medial frontal gyrus, WM & 10 \\
\hline & -6 & 62 & -4 & 1,024 & 5.23 & 4.98 & L. medial frontal gyrus, WM & \\
\hline & 24 & -86 & 16 & 1,759 & 5.74 & 5.42 & R. occipital ceneus, WM & ROI4 \\
\hline & 54 & -64 & 6 & 1,759 & 5 & 4.78 & R. middle temporal gyrus, WM & \\
\hline & -56 & -44 & 32 & 523 & 5.22 & 4.98 & L. parietal supramarginal gyrus, WM & \\
\hline & -50 & -38 & 30 & 523 & 4.41 & 4.26 & L. interior parietal lobule, WM & \\
\hline & -58 & -34 & 18 & 523 & 4.4 & 4.25 & L. superior temporal gyrus, WM & \\
\hline & -30 & 2 & -22 & 732 & 4.49 & 4.33 & L. limbic parahippocampal gyrus, GM & 34 \\
\hline & -24 & -4 & -32 & 732 & 3.65 & 3.56 & L. limbic uncus, WM & ROI5 \\
\hline & 50 & -4 & -22 & 779 & 5.34 & 5.09 & R. temporal subgyrus, WM & \\
\hline & -32 & -26 & -22 & 732 & 5.01 & 4.79 & L. limbic parahippocampal gyrus, WM & ROI2 \\
\hline & 40 & 40 & 26 & 408 & 4.71 & 4.52 & R. middle frontal gyrus, GM & 9 \\
\hline & 50 & -6 & -4 & 779 & 4.6 & 4.43 & R. sublobar insula & \\
\hline & 48 & 30 & 28 & 408 & 4.52 & 4.36 & R. middle frontal gyrus, GM & 9 \\
\hline & 50 & -18 & -4 & 779 & 4.66 & 4.49 & R. superior temporal gyrus, GM & 22 \\
\hline & 46 & 46 & 8 & 408 & 4.03 & 3.91 & R. middle frontal gyrus, WM & \\
\hline
\end{tabular}

gm geometric mean, Neg negative, Pos positive, BA Brodmann area, $R$. right, $L$. left, $W M$ white matter, GM gray matter

the right occipital subgyrus and the left medial and superior frontal gyrus for the gmMD; the right middle occipital gyrus for the nMD; the right middle temporal gyrus, the left medial frontal gyrus, the right occipital cuneus, the left parietal supramarginal gyrus, the left limbic parahippocampal gyrus, the left limbic uncus, the right insula, and the right middle frontal gyrus for the pMD.

Similarly, the overlapping regions of increased MD in the $\mathrm{AD}$ patients relative to that of the control subjects
$(\mathrm{AD}>\mathrm{CN})$ included the left limbic parahippocampal gyrus and right middle temporal gyrus. In contrast, the nonoverlapping regions of increased $\mathrm{MD}$ in the $\mathrm{AD}$ patients relative to that of the control subjects included: the left limbic uncus and the left and right temporal subgyrus for the gmMD; the left and right temporal subgyrus and the right sublobar insula for the nMD; the right temporal subgyrus, the right occipital subgyrus, the right occipital cuneus and the right inferior occipital 
Table 3 The significantly different regions when comparing between the $\mathrm{AD}$ and $\mathrm{CN}$ groups $(\mathrm{AD}>\mathrm{CN})$ using the MD with (gmMD) and without (nMD and pMD) minimizing the effects of the background gradients (corrected FDR $(p<0.01)$ as the cluster level; one-way ANOVA tests)

\begin{tabular}{|c|c|c|c|c|c|c|c|c|}
\hline & \multicolumn{3}{|c|}{ Talairach coordinate } & \multirow[t]{2}{*}{ Cluster } & \multirow[t]{2}{*}{$\mathrm{T}$} & \multirow[t]{2}{*}{$\mathrm{Z}$} & \multirow[t]{2}{*}{ Region } & \multirow[t]{2}{*}{ BA ROI\# } \\
\hline & $X$ & $Y$ & $Z$ & & & & & \\
\hline \multirow[t]{7}{*}{$\mathrm{gm}$} & -32 & -24 & -22 & 289 & 5.18 & 4.94 & L. limbic parahippocampal gyrus, WM & ROI2 \\
\hline & -26 & -8 & -28 & 289 & 4.32 & 4.18 & L. limbic uncus, GM, amygdala & \\
\hline & -30 & 0 & -20 & 289 & 4.29 & 4.15 & L. temporal subgyrus, WM & \\
\hline & 50 & -2 & -22 & 157 & 4.99 & 4.78 & R. temporal subgyrus, WM & \\
\hline & 40 & -74 & 28 & 530 & 4.9 & 4.7 & R. middle temporal gyrus, WM & ROI3 \\
\hline & 56 & -64 & 8 & 530 & 4.69 & 4.51 & R. middle temporal gyrus, WM & \\
\hline & 32 & -66 & 34 & 530 & 4.59 & 4.42 & R. temporal subgyrus, WM & \\
\hline \multirow[t]{8}{*}{ Neg } & -32 & -24 & -22 & 415 & 5.47 & 5.19 & L. limbic parahippocampal gyrus, WM & ROI2 \\
\hline & -26 & -8 & -30 & 415 & 4.9 & 4.69 & L. limbic parahippocampal gyrus, WM & \\
\hline & -30 & 0 & -20 & 415 & 4.22 & 4.09 & L. temporal subgyrus, WM & \\
\hline & 50 & -2 & -22 & 294 & 5.09 & 4.86 & R. temporal subgyrus, WM & \\
\hline & 40 & -74 & 28 & 622 & 4.85 & 4.65 & R. middle temporal gyrus, WM & ROI3 \\
\hline & 34 & -66 & 34 & 622 & 4.7 & 4.52 & R. temporal subgyrus, WM & \\
\hline & 48 & -18 & -4 & 294 & 4.4 & 4.24 & R. sublobar insula, GM & 13 \\
\hline & 46 & -60 & 2 & 622 & 4.47 & 4.31 & R. temporal subgyrus, WM & \\
\hline \multirow[t]{8}{*}{ Pos } & -32 & -26 & -22 & 409 & 4.87 & 4.67 & L. limbic parahippocampal gyrus, WM & ROI2 \\
\hline & -28 & -2 & -20 & 409 & 4.45 & 4.29 & L. limbic parahippocampal gyrus, WM & \\
\hline & 42 & -74 & 28 & 1,010 & 5.22 & 4.95 & R. middle temporal gyrus, WM & ROI3 \\
\hline & 54 & -64 & 8 & 1,010 & 5.28 & 5.03 & R. middle temporal gyrus, WM & \\
\hline & 30 & -66 & 34 & 1,010 & 4.81 & 4.62 & R. occipital subgyrus, WM & \\
\hline & 26 & -84 & 16 & 485 & 5.12 & 4.89 & R. occipital cuneus, WM & ROI4 \\
\hline & 30 & -94 & 4 & 485 & 4.73 & 4.55 & R. occipital subgyrus, WM & \\
\hline & 38 & -94 & -2 & 485 & 4.39 & 4.24 & R. inferior occipital gyrus, WM & \\
\hline
\end{tabular}

$R$. right, $L$. left

gyrus for the pMD. Because the gmFA and nFA differences between the $\mathrm{AD}$ patients and the other two groups of subjects were not significant, there were no overlapping regions for the FA measures.

Tables 8 and 9 list the results of ROI analyses for the $\mathrm{MD}$ and the FA, respectively. As we can see in the tables, $M D$ values were significantly different between $A D$ and $\mathrm{MCI}$ or between $\mathrm{AD}$ and $\mathrm{CN}$. There was no significant difference between MCI and $\mathrm{CN}$. Those results were the same as those from VBM analyses.

\section{Discussion}

A new finding of this study is that in $\mathrm{AD}$ patients, the background gradients have a significantly greater effect on the MD than that in the MCI or CN subjects. This finding is consistent with our hypothesis that the elevated iron-rich processes in $\mathrm{AD}$ patients, such as the accumulation of amyloid plaques, induce local variations in magnetic

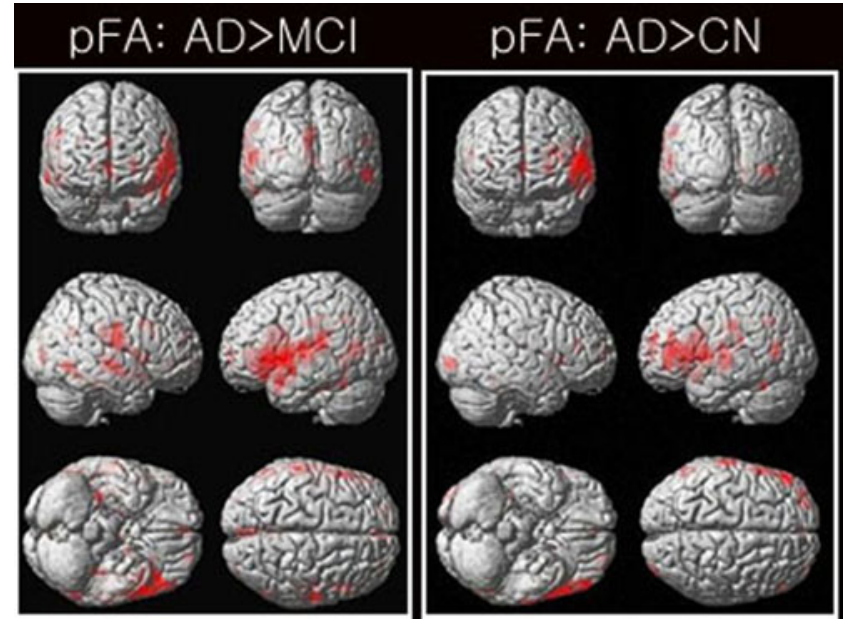

Fig. 2 Results of the voxel-wise comparisons of factional anisotropy (FA; gmFA, $n F A, p F A$ ) among the three $\mathrm{AD}, \mathrm{MCI}$, and $\mathrm{CN}$ groups using one-way ANOVA tests. The pFA maps were not significantly different between the $\mathrm{MCI}$ and $\mathrm{CN}$ groups. The gmFA and nFA maps were not significantly different among the three $\mathrm{AD}, \mathrm{MCI}$, and $\mathrm{CN}$ groups at all 
Table 4 The significantly different regions when comparing between the $\mathrm{AD}$ and $\mathrm{MCI}$ groups $(\mathrm{AD}>\mathrm{MCI})$ using the FA maps with (gmFA) and without (nFA and pFA) minimizing the effects of the background gradients (corrected FDR $(p<0.01)$ as a cluster level; one-way ANOVA tests)

\begin{tabular}{|c|c|c|c|c|c|c|c|c|}
\hline & \multicolumn{3}{|c|}{ Talairach coordinate } & \multirow[t]{2}{*}{ Cluster } & \multirow[t]{2}{*}{$\mathrm{T}$} & \multirow[t]{2}{*}{$\mathrm{Z}$} & \multirow[t]{2}{*}{ Region } & \multirow[t]{2}{*}{ BA ROI\# } \\
\hline & $X$ & $Y$ & $Z$ & & & & & \\
\hline gm & \multicolumn{8}{|c|}{ No significant difference } \\
\hline Neg & \multicolumn{8}{|c|}{ No significant difference } \\
\hline \multirow[t]{14}{*}{ Pos } & -58 & -32 & 14 & 4,975 & 5.95 & 5.6 & L. superior temporal gyrus, WM & ROI6 \\
\hline & -54 & 24 & 8 & 4,975 & 5.89 & 5.56 & L. inferior frontal gyrus, GM & 45 \\
\hline & 52 & -12 & 22 & 1,550 & 5.75 & 5.43 & R. sublobar extranuclear, WM & \\
\hline & 50 & 2 & 10 & 1,550 & 5.46 & 5.18 & R. sublobar insula, WM & \\
\hline & 38 & -24 & 18 & 1,550 & 5.91 & 4.71 & R. sublobar insula, GM & \\
\hline & 34 & -30 & -16 & 383 & 5.51 & 5.23 & R. limbic parahippocampal gyrus, WM & \\
\hline & 50 & -40 & -22 & 383 & 3.9 & 3.79 & R. temporal fusiform gyrus, GM & 13 \\
\hline & 64 & -16 & -6 & 459 & 4.91 & 4.71 & R. superior temporal gyrus, WM & ROI7 \\
\hline & 58 & -26 & -4 & 459 & 5.26 & 4.12 & R. superior temporal gyrus, WM & 37 ROI8 \\
\hline & -2 & -74 & 26 & 761 & 4.83 & 4.63 & L. occipital cuneus, GM & 18 \\
\hline & -2 & -60 & 30 & 761 & 4.65 & 4.47 & L. limbic posterior cingulate & \\
\hline & -2 & -88 & 18 & 761 & 4.47 & 4.31 & L. occipital cuneus, GM & 18 \\
\hline & -56 & -66 & 10 & 404 & 4.72 & 4.53 & L. middle temporal gyrus, WM & \\
\hline & -60 & -58 & 2 & 404 & 4.24 & 4.1 & L. middle temporal gyrus, WM & \\
\hline
\end{tabular}

$R$. right, $L$. left

susceptibility, and these are detectable as regional changes in the background gradients. This finding suggests that the contributions to the background gradients are heterogeneous. Finally, our results from the regular DT-MRI maps using diffusion-sensitizing gradients with positive polarity alone are consistent with the results of the previous DTMRI studies on $\mathrm{AD}, \mathrm{MCI}$, and $\mathrm{CN}$ subjects.

$\mathrm{AD}$ was associated with a systematic pattern of diffusion alternations in the MD maps relative to $\mathrm{MCI}$ and $\mathrm{CN}$ even when the geometrical mean values of diffusivity (gmMD) were computed, which should in principle lead to diminished effects of the background gradients. The increased gmMD values in the $\mathrm{AD}$ subjects were found in the region of the right superior temporal gyrus. A previous DT-MRI study that used single polarity diffusion-encoding gradients reported elevated $\mathrm{MD}$ values in the $\mathrm{AD}$ subjects relative to that in the MCI subjects in the same region [5]. In addition, we also found increased gmMD values in the AD subjects in the left superior frontal gyrus gray matter and left medial frontal gyrus white matter. Furthermore, compared to the $\mathrm{CN}$ subjects, we found increased gmMD values in the $\mathrm{AD}$ subjects in the limbic parahippocampal and right middle temporal gyrus, and the left and right temporal white matter. Those areas were also consistent with those of the previous single polarity DT-MRI studies in AD patients [1, 3, 4, 27]. Furthermore, we found increased gmMD values in the AD subjects involving the left limbic uncus gray matter, and this region has not been reported before in DT-MRI studies. The findings are consistent with our results of comparing the MD maps derived from
Table 5 The significantly different regions when comparing between the $\mathrm{AD}$ and $\mathrm{CN}$ groups $(\mathrm{AD}>\mathrm{CN})$ using the FA maps with (gmFA) and without (nFA and $\mathrm{pFA}$ ) minimizing the effects of the background gradients (corrected FDR $(p<0.01)$ as a cluster level; one-way ANOVA tests)

$R$. right, $L$. left

\begin{tabular}{lllllll}
\hline Talairach coordinate & Cluster & $\mathrm{T}$ & $\mathrm{Z}$ & Region & BA ROI\# \\
\hline & & & & & & \\
\hline
\end{tabular}

gm No significant difference

Neg No significant difference

\begin{tabular}{lllllllll} 
Pos & -48 & 42 & -4 & 2,986 & 6.49 & 6.05 & L. inferior frontal gyrus, WM & \\
& -56 & 22 & 8 & 2,986 & 5.78 & 5.46 & L. inferior frontal gyrus & \\
& -36 & 56 & 16 & 357 & 4.81 & 4.62 & L. superior frontal gyrus, GM & 10 \\
-24 & 56 & 4 & 357 & 4.39 & 4.24 & L. superior frontal gyrus, WM & ROI9 \\
-28 & 54 & 24 & 357 & 4.29 & 4.15 & L. superior frontal gyrus, WM & ROI10 \\
\hline
\end{tabular}


Table 6 Summaries of the common and different regions from Tables 2 and 3 for the mean diffusivity (MD)

\begin{tabular}{|c|c|}
\hline & Region \\
\hline \multicolumn{2}{|c|}{ Common regions $(\mathrm{AD}>\mathrm{MCI})$} \\
\hline ROI1 & $\begin{array}{l}\text { R. superior temporal gyrus, WM } \\
\text { R. temporal subgyrus, WM } \\
\text { R. superior temporal gyrus, GM }\end{array}$ \\
\hline ROI2 & L. limbic parahippocampal gyrus, WM \\
\hline \multicolumn{2}{|c|}{ Different regions $(\mathrm{AD}>\mathrm{MCI})$} \\
\hline gmMD & $\begin{array}{l}\text { R. occipital subgyrus, WM } \\
\text { L. superior frontal gyrus, GM } \\
\text { L. medial frontal gyrus, WM }\end{array}$ \\
\hline nMD & R. middle occipital gyrus, WM \\
\hline pMD (ROI3) & $\begin{array}{l}\text { R. middle temporal gyrus, WM } \\
\text { R. and L. medial frontal gyrus, GM and WM }\end{array}$ \\
\hline ROI4 & $\begin{array}{l}\text { R. occipital ceneus, WM } \\
\text { L. parietal supramarginal gyrus, WM } \\
\text { L. interior parietal lobule, WM } \\
\text { L. limbic parahippocampal gyrus, GM }\end{array}$ \\
\hline ROI5 & $\begin{array}{l}\text { L. limbic uncus, WM } \\
\text { R. sublobar insula }\end{array}$ \\
\hline \multicolumn{2}{|c|}{ Common regions $(\mathrm{AD}>\mathrm{CN})$} \\
\hline $\begin{array}{l}\text { ROI2 } \\
\text { ROI3 }\end{array}$ & $\begin{array}{l}\text { L. limbic parahippocampal gyrus, WM } \\
\text { R. middle temporal gyrus, WM }\end{array}$ \\
\hline \multicolumn{2}{|c|}{ Different regions $(\mathrm{AD}>\mathrm{CN})$} \\
\hline gmMD & $\begin{array}{l}\text { L. limbic uncus, GM, Amygdala } \\
\text { R. and L. temporal subgyrus, WM }\end{array}$ \\
\hline nMD & $\begin{array}{l}\text { R. and L. temporal subgyrus, WM } \\
\text { R. sublobar insula, GM }\end{array}$ \\
\hline pMD & R. temporal subgyrus, WM \\
\hline ROI4 & $\begin{array}{l}\text { R. occipital cuneus, WM } \\
\text { R. occipital subgyrus, WM } \\
\text { R. inferior occipital gyrus, WM }\end{array}$ \\
\hline
\end{tabular}

$R$. right, $L$. left

the diffusion-sensitizing gradients with different polarity, and the findings further support our hypothesis that intrinsic susceptibility variations of the brain contribute to the regional diffusion abnormalities in $\mathrm{AD}$ patients.

One of our findings was that the regional distribution of elevated gmMD values in the AD patients did not completely overlap with the corresponding pattern of $\mathrm{nMD}$ and $\mathrm{pMD}$ variations in the AD patients. The regional difference could reflect the heterogeneity of AD pathology or different stages of the disease, as well as the variations in the processes leading to local variations in magnetic susceptibility. For example, the regions where all three diffusion measurements (pMD, nMD, and gmMD) show significant alterations might be related to alterations primarily in the cell density that alter the MD, but they do not substantially alter the magnet susceptibility. On the
Table 7 Summaries of the common and different regions from Tables 2 and 3 for fractional anisotropy (FA)

\begin{tabular}{|c|c|}
\hline & Region \\
\hline \multicolumn{2}{|c|}{$\begin{array}{l}\text { Common regions }(\mathrm{AD}> \\
\mathrm{MCI})\end{array}$} \\
\hline & No common region \\
\hline \multicolumn{2}{|c|}{ Different regions $(\mathrm{AD}>\mathrm{MCI})$} \\
\hline \multirow[t]{9}{*}{ pFA (ROI 6,7,8) } & $\begin{array}{l}\text { R. and L. superior temporal gyrus, } \\
\text { WM }\end{array}$ \\
\hline & L. inferior frontal gyrus, GM \\
\hline & R. sublobar extranuclear, WM \\
\hline & R. sublobar insula, GM and WM \\
\hline & $\begin{array}{l}\text { R. limbic parahippocampal gyrus, } \\
\text { WM }\end{array}$ \\
\hline & R. temporal fusiform gyrus, GM \\
\hline & L. occipital cuneus, GM \\
\hline & L. limbic posterior cingulate \\
\hline & L. middle temporal gyrus, WM \\
\hline \multicolumn{2}{|c|}{$\begin{array}{l}\text { Common regions }(\mathrm{AD}> \\
\mathrm{CN})\end{array}$} \\
\hline & No common region \\
\hline \multicolumn{2}{|c|}{ Different regions $(\mathrm{AD}>\mathrm{CN})$} \\
\hline $\mathrm{pFA}$ & L. inferior frontal gyrus, WM \\
\hline ROI 9,10 & $\begin{array}{l}\text { L. superior frontal gyrus, GM and } \\
\text { WM }\end{array}$ \\
\hline
\end{tabular}

$R$. right, $L$. left

other hand, the regions without a significant change in the gmMD, but that are with significant changes in the nMD and pMD, could mainly reflect the characteristic local magnetic susceptibility variations related to the paramagnetic effects from iron-rich processes involving the oligodentrocytes and amyloid plaques. The sensitivity differences between in the various MD maps cannot explain the regional discordance between the changes in the pMD and nMD. We cannot directly compare the sensitivity difference between the gmMD maps and the pMD or nMD maps because the gmMD map is derived by averaging two DT-MRI signals and generally has a higher signal-to-noise ratio than the individual pMD and nMD, which are based on a single DT-MRI signal. Therefore, the geometrically calculated MD (gmMD) in the $\mathrm{AD}$ or MCI patients may be better to differentiate accurately between the groups as compared to that obtained with the single polarity.

We tested our concepts in water phantom before we applied in human brain. The mean ADC value or average tensor components or mean eigenvalue is different about $0.00004 \mathrm{~mm}^{2} / \mathrm{s}$, and the FA value is different about 0.00188 between the positive and negative diffusion gradients. This means that our human results should be clearly shown reliable measurements of DTI data without absence of any 
Table 8 The ROI data and results of the corresponding statistical test for the mean diffusivity (MD) values

\begin{tabular}{|c|c|c|c|c|c|c|c|c|}
\hline \multirow[t]{2}{*}{ ROI } & & & \multicolumn{3}{|l|}{ Subjects } & \multicolumn{3}{|l|}{$p$ value } \\
\hline & & & $\mathrm{AD}$ & MCI & $\mathrm{CN}$ & AD-MCI & $\mathrm{AD}-\mathrm{CN}$ & $\mathrm{MCI}-\mathrm{CN}$ \\
\hline \multirow{6}{*}{$\begin{array}{l}\text { Superior temporal gyrus, ROI1, } \\
\mathrm{X} \pm 58, \mathrm{Y}-6, \mathrm{z}-4\end{array}$} & $\mathrm{Rt}$ & $\mathrm{gm}$ & $1.045 \pm 0.156$ & $0.875 \pm 0.072$ & $0.908 \pm 0.084$ & 0.00026 & 0.00490 & 0.22578 \\
\hline & $\mathrm{Rt}$ & neg & $1.049 \pm 0.161$ & $0.870 \pm 0.076$ & $0.907 \pm 0.091$ & 0.00022 & 0.00500 & 0.21123 \\
\hline & $\mathrm{Rt}$ & pos & $1.042 \pm 0.153$ & $0.879 \pm 0.069$ & $0.909 \pm 0.079$ & 0.00030 & 0.00450 & 0.24897 \\
\hline & $\mathrm{Lt}$ & $\mathrm{gm}$ & $1.023 \pm 0.155$ & $0.911 \pm 0.117$ & $0.955 \pm 0.099$ & 0.02489 & 0.15434 & 0.25234 \\
\hline & $\mathrm{Lt}$ & neg & $1.027 \pm 0.163$ & $0.917 \pm 0.127$ & $0.960 \pm 0.108$ & 0.03755 & 0.18508 & 0.30315 \\
\hline & $\mathrm{Lt}$ & pos & $1.022 \pm 0.150$ & $0.907 \pm 0.110$ & $0.954 \pm 0.092$ & 0.01635 & 0.13488 & 0.19277 \\
\hline \multirow{6}{*}{$\begin{array}{l}\text { Limbic parahippocampal gyrus, ROI } 2 \text {, } \\
\quad \mathrm{X} \pm 32, \mathrm{y}-24, \mathrm{z}-22\end{array}$} & $\mathrm{Rt}$ & gm & $0.100 \pm 0.111$ & $0.891 \pm 0.071$ & $0.882 \pm 0.060$ & 0.00187 & 0.00091 & 0.69383 \\
\hline & $\mathrm{Rt}$ & neg & $0.933 \pm 0.111$ & $0.877 \pm 0.074$ & $0.875 \pm 0.061$ & 0.00112 & 0.00088 & 0.93385 \\
\hline & Rt & pos & $1.007 \pm 0.111$ & $0.906 \pm 0.074$ & $0.890 \pm 0.058$ & 0.00395 & 0.00088 & 0.49030 \\
\hline & $\mathrm{Lt}$ & gm & $1.036 \pm 0.116$ & $0.879 \pm 0.060$ & $0.882 \pm 0.075$ & 0.00002 & 0.00012 & 0.92913 \\
\hline & $\mathrm{Lt}$ & neg & $1.031 \pm 0.111$ & $0.868 \pm 0.058$ & $0.870 \pm 0.074$ & 0.00001 & 0.00005 & 0.94042 \\
\hline & $\mathrm{Lt}$ & pos & $1.036 \pm 0.118$ & $0.890 \pm 0.062$ & $0.893 \pm 0.078$ & 0.00008 & 0.00039 & 0.90633 \\
\hline \multirow{6}{*}{$\begin{array}{l}\text { Middle temporal gyrus, } \\
\text { ROI } 3, X \pm 40, y-74, \text { z } 28\end{array}$} & $\mathrm{Rt}$ & gm & $0.963 \pm 0.099$ & $0.837 \pm 0.062$ & $0.832 \pm 0.058$ & 0.00011 & 0.00009 & 0.78235 \\
\hline & $\mathrm{Rt}$ & neg & $0.963 \pm 0.108$ & $0.832 \pm 0.062$ & $0.836 \pm 0.062$ & 0.00013 & 0.00034 & 0.89277 \\
\hline & $\mathrm{Rt}$ & pos & $0.966 \pm 0.105$ & $0.843 \pm 0.064$ & $0.829 \pm 0.054$ & 0.00025 & 0.00008 & 0.49316 \\
\hline & $\mathrm{Lt}$ & gm & $0.944 \pm 0.115$ & $0.852 \pm 0.059$ & $0.875 \pm 0.078$ & 0.00590 & 0.05985 & 0.33837 \\
\hline & $\mathrm{Lt}$ & neg & $0.944 \pm 0.118$ & $0.846 \pm 0.065$ & $0.871 \pm 0.077$ & 0.00511 & 0.05068 & 0.30961 \\
\hline & $\mathrm{Lt}$ & pos & $0.947 \pm 0.114$ & $0.859 \pm 0.054$ & $0.879 \pm 0.081$ & 0.01048 & 0.14942 & 0.23174 \\
\hline \multirow{6}{*}{$\begin{array}{l}\text { Occipital cuneus, ROI } 4, \mathrm{X} \pm 26 \text {, } \\
\text { y }-84, \text { z } 16\end{array}$} & $\mathrm{Rt}$ & gm & $0.948 \pm 0.090$ & $0.829 \pm 0.051$ & $0.839 \pm 0.058$ & 0.00004 & 0.00035 & 0.61712 \\
\hline & $\mathrm{Rt}$ & neg & $0.934 \pm 0.082$ & $0.828 \pm 0.059$ & $0.839 \pm 0.060$ & 0.00015 & 0.00085 & 0.59032 \\
\hline & $\mathrm{Rt}$ & pos & $0.962 \pm 0.106$ & $0.830 \pm 0.046$ & $0.838 \pm 0.057$ & 0.00004 & 0.00031 & 0.65473 \\
\hline & $\mathrm{Lt}$ & gm & $0.924 \pm 0.103$ & $0.853 \pm 0.080$ & $0.879 \pm 0.063$ & 0.03507 & 0.15081 & 0.31487 \\
\hline & $\mathrm{Lt}$ & neg & $0.920 \pm 0.104$ & $0.853 \pm 0.087$ & $0.873 \pm 0.063$ & 0.05182 & 0.13221 & 0.45565 \\
\hline & $\mathrm{Lt}$ & pos & $0.927 \pm 0.107$ & $0.853 \pm 0.075$ & $0.884 \pm 0.064$ & 0.02715 & 0.18312 & 0.21208 \\
\hline \multirow{6}{*}{$\begin{array}{l}\text { Limbic uncus, ROI } 5, X \pm 24 \text {, } \\
y-4, z-32\end{array}$} & $\mathrm{Rt}$ & $\mathrm{gm}$ & $1.047 \pm 0.142$ & $0.950 \pm 0.103$ & $0.928 \pm 0.066$ & 0.03111 & 0.00532 & 0.46841 \\
\hline & $\mathrm{Rt}$ & neg & $1.040 \pm 0.165$ & $0.933 \pm 0.111$ & $0.918 \pm 0.081$ & 0.03475 & 0.01353 & 0.65553 \\
\hline & $\mathrm{Rt}$ & pos & $1.053 \pm 0.135$ & $0.967 \pm 0.100$ & $0.943 \pm 0.065$ & 0.04522 & 0.00692 & 0.42162 \\
\hline & $\mathrm{Lt}$ & $\mathrm{gm}$ & $1.055 \pm 0.118$ & $0.962 \pm 0.071$ & $0.912 \pm 0.097$ & 0.00053 & 0.00090 & 0.61871 \\
\hline & $\mathrm{Lt}$ & neg & $1.053 \pm 0.119$ & $0.912 \pm 0.074$ & $0.899 \pm 0.099$ & 0.00023 & 0.00049 & 0.66891 \\
\hline & $\mathrm{Lt}$ & pos & $1.062 \pm 0.124$ & $0.939 \pm 0.069$ & $0.923 \pm 0.098$ & 0.00118 & 0.00166 & 0.57150 \\
\hline
\end{tabular}

Rt right, $L t$ left, $A D$ Alzheimer's disease, $M C I$ mild cognitive impairment, $C N$ cognitive normal, $G m$ geometric mean, neg negative, $p$ positive The ROIs are listed in the table above. The significant level was used with $p=0.016$ ( $p=0.05 / 3$ times repeated) because we repeated the same tasks three times among the three groups

systematic bias due to the different gradient settings (positive vs negative).

In voxel-wise comparisons of human brain data, the results from geometric mean appear highly similar to those from negative gradients, while positive gradients show very different results. We may explain the reason as the following. The largest contribution of cross terms tends to be when the applied diffusion gradient and the intrinsic background magnetic field gradient are in the same direction and sign as the frequency-encoding direction. With the used TE value, this would mean that we would get more positive pixels for positive diffusion and read gradients than for negative diffusion and positive read gradients. This is supported by getting comparable diffusion rates for all gradient directions on an isotropic phantom experiment with the same acquisition parameters.

Our DTI findings using diffusion-sensitizing gradients with the single positive polarity are largely consistent with the findings of the previous DT-MRI studies that used a single gradient polarity [1, 3-5, 27]. Specifically, our finding in $\mathrm{AD}$ patients of increased $\mathrm{pMD}$ values in the right middle temporal gyrus white matter and the left superior temporal gyrus white matter, as compared to that of the MCI patients, are consistent with a previous report 
Table 9 The ROI data and results of the corresponding statistical test for the fractional anisotropy (FA) values

\begin{tabular}{|c|c|c|c|c|c|c|c|c|}
\hline \multirow[t]{2}{*}{ ROI } & & & \multicolumn{3}{|l|}{ Subjects } & \multicolumn{3}{|l|}{$p$ value } \\
\hline & & & $\mathrm{AD}$ & MCI & $\mathrm{CN}$ & AD-MCI & $\mathrm{AD}-\mathrm{CN}$ & $\mathrm{MCI}-\mathrm{CN}$ \\
\hline \multirow{6}{*}{$\begin{array}{l}\text { Superior temporal gyrus, ROI } 6 \text {, } \\
\mathrm{x} \pm 58, \mathrm{y}-32, \mathrm{z}+14\end{array}$} & Rt & $\mathrm{gm}$ & $0.238 \pm 0.015$ & $0.225 \pm 0.014$ & $0.233 \pm 0.034$ & 0.02207 & 0.65877 & 0.35914 \\
\hline & Rt & neg & $0.293 \pm 0.022$ & $0.269 \pm 0.014$ & $0.280 \pm 0.036$ & 0.00077 & 0.24791 & 0.23248 \\
\hline & Rt & pos & $0.292 \pm 0.027$ & $0.269 \pm 0.016$ & $0.276 \pm 0.046$ & 0.00467 & 0.25159 & 0.54348 \\
\hline & $\mathrm{Lt}$ & $\mathrm{gm}$ & $0.227 \pm 0.016$ & $0.204 \pm 0.014$ & $0.213 \pm 0.016$ & 0.00014 & 0.02329 & 0.10851 \\
\hline & $\mathrm{Lt}$ & neg & $0.282 \pm 0.026$ & $0.250 \pm 0.017$ & $0.266 \pm 0.021$ & 0.00024 & 0.07557 & 0.02310 \\
\hline & $\mathrm{Lt}$ & pos & $0.296 \pm 0.027$ & $0.255 \pm 0.020$ & $0.267 \pm 0.023$ & 0.00002 & 0.00341 & 0.12132 \\
\hline \multirow{6}{*}{$\begin{array}{l}\text { Superior temporal gyrus, ROI } 7 \text {, } \\
\quad \mathrm{x} \pm 64, \mathrm{y}-16, \mathrm{z}-6\end{array}$} & Rt & gm & $0.212 \pm 0.023$ & $0.190 \pm 0.013$ & $0.194 \pm 0.012$ & 0.00105 & 0.00807 & 0.32091 \\
\hline & Rt & neg & $0.264 \pm 0.028$ & $0.237 \pm 0.014$ & $0.246 \pm 0.020$ & 0.00137 & 0.04480 & 0.18303 \\
\hline & Rt & pos & $0.271 \pm 0.036$ & $0.236 \pm 0.015$ & $0.244 \pm 0.018$ & 0.00062 & 0.01191 & 0.14461 \\
\hline & $\mathrm{Lt}$ & $\mathrm{gm}$ & $0.120 \pm 0.017$ & $0.190 \pm 0.016$ & $0.181 \pm 0.019$ & 0.10116 & 0.00693 & 0.13342 \\
\hline & $\mathrm{Lt}$ & neg & $0.251 \pm 0.015$ & $0.240 \pm 0.021$ & $0.233 \pm 0.029$ & 0.12077 & 0.04121 & 0.40239 \\
\hline & $\mathrm{Lt}$ & pos & $0.265 \pm 0.036$ & $0.238 \pm 0.015$ & $0.229 \pm 0.021$ & 0.00667 & 0.00202 & 0.18436 \\
\hline \multirow{6}{*}{$\begin{array}{l}\text { Superior temporal gyrus, ROI } 8 \\
\quad \mathrm{x} \pm 58, \mathrm{y}-26, \mathrm{z}-4\end{array}$} & Rt & gm & $0.231 \pm 0.023$ & $0.210 \pm 0.016$ & $0.217 \pm 0.234$ & 0.00477 & 0.11993 & 0.29256 \\
\hline & Rt & neg & $0.276 \pm 0.026$ & $0.254 \pm 0.021$ & $0.265 \pm 0.030$ & 0.01128 & 0.31982 & 0.19190 \\
\hline & Rt & pos & $0.284 \pm 0.032$ & $0.249 \pm 0.013$ & $0.262 \pm 0.028$ & 0.00018 & 0.05107 & 0.08649 \\
\hline & $\mathrm{Lt}$ & gm & $0.223 \pm 0.026$ & $0.218 \pm 0.020$ & $0.220 \pm 0.020$ & 0.55759 & 0.67971 & 0.85453 \\
\hline & $\mathrm{Lt}$ & neg & $0.270 \pm 0.020$ & $0.266 \pm 0.026$ & $0.268 \pm 0.029$ & 0.62074 & 0.84838 & 0.80622 \\
\hline & $\mathrm{Lt}$ & pos & $0.281 \pm 0.030$ & $0.263 \pm 0.018$ & $0.269 \pm 0.026$ & 0.03818 & 0.22205 & 0.44215 \\
\hline \multirow{6}{*}{$\begin{array}{l}\text { Superior frontal gyrus, ROI 9, } \\
\mathrm{x} \pm 24, \mathrm{y}+56, \mathrm{z}+4\end{array}$} & Rt & $\mathrm{gm}$ & $0.253 \pm 0.027$ & $0.255 \pm 0.026$ & $0.254 \pm 0.033$ & 0.87967 & 0.93917 & 0.95524 \\
\hline & Rt & neg & $0.303 \pm 0.022$ & $0.293 \pm 0.023$ & $0.301 \pm 0.033$ & 0.24157 & 0.90915 & 0.40468 \\
\hline & Rt & pos & $0.311 \pm 0.030$ & $0.302 \pm 0.036$ & $0.295 \pm 0.030$ & 0.47368 & 0.15143 & 0.51314 \\
\hline & $\mathrm{Lt}$ & gm & $0.268 \pm 0.025$ & $0.244 \pm 0.027$ & $0.247 \pm 0.025$ & 0.01453 & 0.02980 & 0.73554 \\
\hline & $\mathrm{Lt}$ & neg & $0.311 \pm 0.026$ & $0.289 \pm 0.034$ & $0.302 \pm 0.030$ & 0.05134 & 0.39898 & 0.24469 \\
\hline & $\mathrm{Lt}$ & pos & $0.347 \pm 0.045$ & $0.303 \pm 0.034$ & $0.298 \pm 0.030$ & 0.00298 & 0.00125 & 0.67386 \\
\hline \multirow{6}{*}{$\begin{array}{l}\text { Superior frontal gyrus, ROI } 10 \text {, } \\
\quad \mathrm{x} \pm 28, \mathrm{y}+58, \mathrm{z}+24\end{array}$} & Rt & gm & $0.205 \pm 0.027$ & $0.188 \pm 0.027$ & $0.182 \pm 0.028$ & 0.08842 & 0.03057 & 0.54784 \\
\hline & Rt & neg & $0.249 \pm 0.042$ & $0.235 \pm 0.032$ & $0.235 \pm 0.033$ & 0.28368 & 0.30211 & 0.98821 \\
\hline & Rt & pos & $0.279 \pm 0.046$ & $0.248 \pm 0.041$ & $0.239 \pm 0.037$ & 0.04714 & 0.01125 & 0.49593 \\
\hline & $\mathrm{Lt}$ & $\mathrm{gm}$ & $0.203 \pm 0.027$ & $0.178 \pm 0.027$ & $0.168 \pm 0.042$ & 0.00985 & 0.00989 & 0.44181 \\
\hline & $\mathrm{Lt}$ & neg & $0.258 \pm 0.028$ & $0.229 \pm 0.036$ & $0.223 \pm 0.052$ & 0.01580 & 0.02985 & 0.73585 \\
\hline & $\mathrm{Lt}$ & pos & $0.272 \pm 0.039$ & $0.241 \pm 0.045$ & $0.221 \pm 0.057$ & 0.05028 & 0.00800 & 0.26447 \\
\hline
\end{tabular}

$R t$ right, $L t$ left, $A D$ Alzheimer's disease, $M C I$ mild cognitive impairment, $C N$ cognitive normal, $G m$ geometric mean, neg negative, $p$ positive The ROIs are listed in the table above. The significant level was used with $p=0.016$ ( $p=0.05 / 3$ times repeated) because we repeated the same tasks three times among the three groups

[5]. Similarly, our results in AD patients of increased pMD values in the limbic parahippocampal gyrus white matter, the right temporal gyrus white matter, and the right occipital white matter are consistent with the results of the previous studies [1, 3, 4, 27].

Previous studies have also demonstrated that the background gradient effects in DT-MRI can be amplified due to an interaction (also known as the "cross-term effect") between the external and background gradients. Several diffusion studies have reported significant cross-term effects on the measurements of the apparent diffusion coefficients (ADC) in phantoms [28], rat brain [14], and pig spinal cord [29]. Indeed, several studies have shown that cross-term effects can also depend, at least in part, on the types of sequence and the external gradient patterns [30, 31 , and these effects can in principle be minimized by the combined use of a multispin-echo preparation and pulse gradients for diffusion encoding [32] or the combined use of asymmetric bipolar diffusion-encoding gradients and twice refocused spin-echo preparation [22]. A further class of experimental strategies that also accounts for the presence of field inhomogeneities is based on the use of bipolar gradient pluses [22, 33, 34]. However, some ADC studies have found no detectable cross-term effects [35], 
including a DT-MRI study in normal young human brain [36]. Our results generalize the cross-term findings by demonstrating that the effect can substantially vary between different brain conditions. Our results in AD patients that not all the variations in the pMD or nMD maps were also present in the gmMD maps emphasize the importance of accounting for the background gradients when comparing the DT-MRI data between groups with different brain conditions. In particular, the findings indicate that a bias toward increased MD variability can be introduced if the background gradients are not considered. For the FA index, there were no differences between the positive and negative acquisitions. This is not surprising because FA is derived by taking the ratios of the eigenvalues. The sensitivity to detect an effect on FA is substantially diminished as compared to that of MD, which is simply the average of the eigenvalues.

Finally, although some previous investigators [2, 37, 38] have found significant differences of FA and MD between the MCI and CN patients, we did not find any significant differences for either FA or MD between these two groups. One explanation is that the MCI subjects in this study were only mildly impaired, as was reflected by their average MMSE score, which did not significantly differ from the score of the normal subjects. Another possibility is that our MCI group included only a few subjects with preclinical $\mathrm{AD}$ pathology as compared to that of the MCI groups in the other previous studies.

In general, the FA values in the white matter can be contaminated by signal from CSF spaces, which are more pronounced in $\mathrm{AD}$ than in $\mathrm{CN}$ subject. This will support increased MD and decreased FA values which showed several previous papers. However, in our study, we use the CSF-suppressed DTI method to minimize partial volume effect. Increased MD values in a voxel may be not directly related to CSF contamination. We think that this may be related to loss of integrity of neuronal structure. In this case, FA value can also decrease or increase dependent on the types of neuronal loss in the voxel. Previous findings of decreased FA in AD may be due to (1) neuronal loss in the voxel (microstructural changes) or (2) increased contributions of CSF signals caused by loss of cortical GM (atrophy; macroscopic changes) because many of previous studies did not use an inversion-recovery DTI sequence. A lot of CSF signals may be contributed to decreased FA values in $\mathrm{AD}$ in the previous findings. However, previous and our findings of increased FA in $\mathrm{AD}$ may be due to loss selectively in a certain direction rather than every neuron in the voxel (selective neuronal loss and microstructural changes). In addition, our finding of increased FA in $\mathrm{AD}$ may be additionally due to less contribution of CSF signals. If brain atrophy is contributed to FA changes, then our finding may be unrelated to microstructural alternation. The recent paper has shown increased FA values in those patients, although they did not use any kinds of CSF suppression techniques. Teipel et al. found increased FA values in $\mathrm{AD}$ patients compared with elderly controls [39]. This increased FA values in AD may be associated with a decrease in crossing fibers or other nonparallel organization [40]. Teipel et al. [39] also found decreased FA values in AD patients compared with elderly control, but not in the present study. The largest difference between two studies is the selection of a statistical threshold. Teipel et al. [39] used uncorrected $p$ value $=0.01$, but we used FDR-corrected $p$ value $=0.01$ to consider multiple comparisons to reduce the false-positive findings. When we reinvestigated the results with the uncorrected $p$ value $=0.01$, then we also found decreased FA values in $\mathrm{AD}$ patients compared with elderly control in the temporal and frontal lobes which were similar findings from Teipel et al. [39]. In addition, there is usually more gray matter loss in $\mathrm{AD}$ than in elderly normal subjects. Within a voxel which composes both gray matter and white matter, FA in our study can also be increased when there is gray matter loss without changing white matter volume because we acquired DTI data with suppressing signals from the brain atrophy. In this case, the relative contribution of white matter in the voxel can be increased. In general, people have been found increased MD and decreased FA in AD compared with normal. This decreased FA may only represent increased atrophy in a certain voxel because people have analyzed their FA data without correcting the atrophy contribution. In this study, we used an inversion-recovery DT-MRI sequence, which minimize the CSF contamination in a voxel. During FA analysis, we may find tissue alternations in the voxel, independent on the contributions of brain atrophy, which usually happens in gray matter. Therefore, it may be very important to correct the brain atrophy before analyzing FA maps, especially obtained FA maps in patients with AD.

In this study, $\mathrm{MD}$ values in some ROIs are higher in $\mathrm{CN}$ than in MCI. FA values in some ROIs are higher in MCI than in $\mathrm{CN}$. We cannot clearly explain why this happens. However, we may think as the following: Although we divided the subject groups as $\mathrm{MCI}$ and $\mathrm{CN}$ based on the full neuropsychologic tests, the MMSE score does not significantly differ between two groups. In addition, although there is no significant difference of age between two groups, the MCI group is relatively younger than the $\mathrm{CN}$ group. As we found the results based on both the voxelwise and the ROI-based comparisons, there were no significant differences any indices between two groups. Although several previous studies found increased MD values and decreased $F A$ values in $A D$ compared with $C N$, some studies also did not found any differences between $\mathrm{MCI}$ and $\mathrm{CN}$ groups for both indices [40]. In this case, the pathological changes in some areas in $\mathrm{AD}$ may not yet 
affect individuals with MCI. Furthermore, as we can see in Table 8, MD values in MCI are higher than those in $\mathrm{CN}$ in the right limbic parahippocampal gyrus, in the left middle temporal gyrus, and in the right and left limbic uncus, as expected. In addition, as we can see in Table 9, FA values in most ROIs are higher in $\mathrm{CN}$ than those in MCI, especially in the right and left superior temporal gyrus, as expected. The pathological changes in those areas may start to affect individuals with MCI. This may indicate regional variations of MD and FA values in patients with MCI compared with $\mathrm{CN}$ subjects.

Several limitations of our study ought to be mentioned. Firstly, none of the AD patient had a definite diagnosis confirmed by autopsy. Therefore, the DTI alterations in the $\mathrm{AD}$ patients might not be related to $\mathrm{AD}$ at all. Another limitation is that we did not investigate the spatially variant background gradients for the higher order diffusion effects, such as kurtosis. Therefore, the contributions from these other effects might have biased our results. Another technical limitation is that the geometrical average maps, which are derived by averaging two signals, have a higher SNR than do the individual maps of diffusivity without averaging, and this leads to differences in sensitivity. Therefore, the findings of regional discrepancies between these maps are difficult to interpret. Finally, in this study, we mainly used a voxel-based analysis of the DTI indices of both MD and FA maps. Currently, the voxelbased analysis is still under investigating in optimization processing of DT-MRI data. Although the voxel-based analysis can be used a whole brain investigation without having a specific hypothesis, the results may be altered by several factors, such as a spatial normalization of DTMRI data acquired with an EPI sequence, a coregistration between anatomic magnetic resonance images and maps of DTI indices, a filter size of smoothing kernel, and/or registration of individual MD or FA maps onto a common space. Therefore, optimizations of the preprocessing and postprocessing steps are required to minimize any errors during processing $[41,42]$.

\section{Conclusions}

Accurate DT-MRI measurements require considering the effects from background gradients, and especially in patients with pathological brain conditions such as AD. Furthermore, geometric average diffusion measures (e.g., gmMD) can be useful to minimize the intrinsic local magnetic susceptibility variations in brain tissue. As we demonstrated for the case of $\mathrm{AD}$, these maps may provide complementary information to the standard DTI maps.
Acknowledgments We thank Dr. Michal Neeman at Weizmann Institute in Rehovot, Israel, for his valuable comments. This research was supported by a grant of the Korean Health 21 R\&D Project, Ministry of Health and Welfare, Republic of Korea (A062284).

Conflict of interest We declare that we have no conflict of interest.

Open Access This article is distributed under the terms of the Creative Commons Attribution Noncommercial License which permits any noncommercial use, distribution, and reproduction in any medium, provided the original author(s) and source are credited.

\section{References}

1. Duan JH, Wang HQ, Xu J, Lin X, Chen SQ, Kang Z, Yao ZB (2006) White matter damage of patients with Alzheimer's disease correlated with the decreased cognitive function. Surg Radiol Anat 28:150-156

2. Medina D, DeToledo-Morrell L, Urresta F, Gabrieli JD, Moseley M, Fleischman D, Bennett DA, Leurgans S, Turner DA, Stebbins GT (2006) White matter changes in mild cognitive impairment and $\mathrm{AD}$ : a diffusion tensor imaging study. Neurobiol Aging 27:663-672

3. Naggara O, Oppenheim C, Rieu D, Raoux N, Rodrigo S, Dalla Barba G, Meder JF (2006) Diffusion tensor imaging in early Alzheimer's disease. Psychiatry Res 146:243-249

4. Rose SE, Janke AL, Chalk JB (2008) Gray and white matter changes in Alzheimer's disease: a diffusion tensor imaging study. J Magn Reson Imaging 27:20-26

5. Stahl R, Dietrich O, Teipel SJ, Hampel H, Reiser MF, Schoenberg SO (2007) White matter damage in Alzheimer disease and mild cognitive impairment: assessment with diffusion-tensor MR imaging and parallel imaging techniques. Radiology 243:483-492

6. Zhang Y, Schuff N, Jahng GH, Bayne W, Mori S, Schad L, Mueller S, Du AT, Kramer JH, Yaffe K, Chui H, Jagust WJ, Miller BL, Weiner MW (2007) Diffusion tensor imaging of cingulum fibers in mild cognitive impairment and Alzheimer disease. Neurology 68:13-19

7. Chua TC, Wen W, Slavin MJ, Sachdev PS (2008) Diffusion tensor imaging in mild cognitive impairment and Alzheimer's disease: a review. Curr Opin Neurol 21:83-92

8. Nakata Y, Sato N, Abe O, Shikakura S, Arima K, Furuta N, Uno M, Hirai S, Masutani Y, Ohtomo K, Aoki S (2008) Diffusion abnormality in posterior cingulate fiber tracts in Alzheimer's disease: tract-specific analysis. Radiat Med 26:466-473

9. Mielke MM, Kozauer NA, Chan KC, George M, Toroney J, Zerrate M, Bandeen-Roche K, Wang MC, Vanzijl P, Pekar JJ, Mori S, Lyketsos CG, Albert M (2009) Regionally-specific diffusion tensor imaging in mild cognitive impairment and Alzheimer's disease. Neuroimage 46:47-55

10. Huang J, Friedland RP, Auchus AP (2007) Diffusion tensor imaging of normal-appearing white matter in mild cognitive impairment and early Alzheimer disease: preliminary evidence of axonal degeneration in the temporal lobe. AJNR Am J Neuroradiol 28:1943-1948

11. Roth AD, Ramirez G, Alarcon R, Von Bernhardi R (2005) Oligodendrocytes damage in Alzheimer's disease: beta amyloid toxicity and inflammation. Biol Res 38:381-387

12. Schmitz T, Chew LJ (2008) Cytokines and myelination in the central nervous system. Scientific World J 8:1119-1147 
13. Bartzokis G (2004) Age-related myelin breakdown: a developmental model of cognitive decline and Alzheimer's disease. Neurobiol Aging 25:5-18, Author reply 49-62

14. Does MD, Zhong J, Gore JC (1999) In vivo measurement of ADC change due to intravascular susceptibility variation. Magn Reson Med 41:236-240

15. Connor JR, Menzies SL, St Martin SM, Mufson EJ (1992) A histochemical study of iron, transferrin, and ferritin in Alzheimer's diseased brains. J Neurosci Res 31:75-83

16. Smith MA, Wehr K, Harris PL, Siedlak SL, Connor JR, Perry G (1998) Abnormal localization of iron regulatory protein in Alzheimer's disease. Brain Res 788:232-236

17. Smith MA, Harris PL, Sayre LM, Perry G (1997) Iron accumulation in Alzheimer disease is a source of redoxgenerated free radicals. Proc Natl Acad Sci USA 94:9866-9868

18. Neeman M, Freyer JP, Sillerud LO (1991) A simple method for obtaining cross-term-free images for diffusion anisotropy studies in NMR microimaging. Magn Reson Med 21:138-143

19. Jara H, Wehrli FW (1994) Determination of background gradients with diffusion MR imaging. J Magn Reson Imaging 4:787-797

20. Petersen RC, Smith GE, Waring SC, Ivnik RJ, Tangalos EG, Kokmen E (1999) Mild cognitive impairment: clinical characterization and outcome. Arch Neurol 56:303-308

21. Hirsch JG, Bock M, Essig M, Schad LR (1999) Comparison of diffusion anisotropy measurements in combination with the flairtechnique. Magn Reson Imaging 17:705-716

22. Reese TG, Heid O, Weisskoff RM, Wedeen VJ (2003) Reduction of eddy-current-induced distortion in diffusion MRI using a twicerefocused spin echo. Magn Reson Med 49:177-182

23. Jones DK, Horsfield MA, Simmons A (1999) Optimal strategies for measuring diffusion in anisotropic systems by magnetic resonance imaging. Magn Reson Med 42:515-525

24. Ashburner J, Friston KJ (1999) Nonlinear spatial normalization using basis functions. Hum Brain Mapp 7:254-266

25. Ashburner J, Friston KJ (2000) Voxel-based morphometry-the methods. Neuroimage 11:805-821

26. Schwartzman A, Dougherty RF, Taylor JE (2008) False discovery rate analysis of brain diffusion direction maps. Ann Appl Stat 2:153-175

27. Fellgiebel A, Wille P, Muller MJ, Winterer G, Scheurich A, Vucurevic G, Schmidt LG, Stoeter P (2004) Ultrastructural hippocampal and white matter alterations in mild cognitive impairment: a diffusion tensor imaging study. Dement Geriatr Cogn Disord 18:101-108

28. Price WS, Stilbs P, Jonsson B, Soderman O (2001) Macroscopic background gradient and radiation damping effects on high-field PGSE NMR diffusion measurements. J Magn Reson 150:49-56

29. Trudeau JD, Dixon WT, Hawkins J (1995) The effect of inhomogeneous sample susceptibility on measured diffusion anisotropy using NMR imaging. J Magn Reson B 108:2230

30. Duh A, Mohoric A, Stepisnik J, Sersa I (2003) The elimination of magnetic susceptibility artifacts in the micro-image of liquid-solid interfaces: internal gradient modulation by the CPMG RF train. $\mathrm{J}$ Magn Reson 160:47-51

31. Sorland GH, Aksnes D, Gjerdaker L (1999) A pulsed field gradient spin-echo method for diffusion measurements in the presence of internal gradients. J Magn Reson 137:397-401

32. van Dusschoten D, Moonen CT, de Jager PA, Van As H (1996) Unraveling diffusion constants in biological tissue by combining Carr-Purcell-Meiboom-Gill imaging and pulsed field gradient NMR. Magn Reson Med 36:907-913

33. Hong DTX (1992) Measuring diffusion in inhomogeneous systems in imaging mode using antisymmetric sensitizing gradients. J Magn Reson 99:561-570

34. Sun PZ, Seland JG, Cory D (2003) Background gradient suppression in pulsed gradient stimulated echo measurements. J Magn Reson 161:168-173

35. Beaulieu C, Allen PS (1996) An in vitro evaluation of the effects of local magnetic-susceptibility-induced gradients on anisotropic water diffusion in nerve. Magn Reson Med 36:39-44

36. Clark CA, Barker GJ, Tofts PS (1999) An in vivo evaluation of the effects of local magnetic susceptibility-induced gradients on water diffusion measurements in human brain. J Magn Reson 141:52-61

37. Rose SE, McMahon KL, Janke AL, O'Dowd B, de Zubicaray G, Strudwick MW, Chalk JB (2006) Diffusion indices on magnetic resonance imaging and neuropsychological performance in amnestic mild cognitive impairment. J Neurol Neurosurg Psychiatry $77: 1122-1128$

38. Cho H, Yang DW, Shon YM, Kim BS, Kim YI, Choi YB, Lee KS, Shim YS, Yoon B, Kim W, Ahn KJ (2008) Abnormal integrity of corticocortical tracts in mild cognitive impairment: a diffusion tensor imaging study. J Korean Med Sci 23:477483

39. Teipel SJ, Stahl R, Dietrich O, Schoenberg SO, Perneczky R, Bokde AL, Reiser MF, Moller HJ, Hampel H (2007) Multivariate network analysis of fiber tract integrity in Alzheimer's disease. Neuroimage 34:985-995

40. Stebbins GT, Murphy CM (2009) Diffusion tensor imaging in Alzheimer's disease and mild cognitive impairment. Behav Neurol 21:39-49

41. Abe O, Takao H, Gonoi W, Sasaki H, Murakami M, Kabasawa H, Kawaguchi H, Goto M, Yamada H, Yamasue H, Kasai K, Aoki S, Ohtomo K (2010) Voxel-based analysis of the diffusion tensor. Neuroradiology 52:699-710

42. Kakeda S, Korogi Y (2010) The efficacy of a voxel-based morphometry on the analysis of imaging in schizophrenia, temporal lobe epilepsy, and Alzheimer's disease/mild cognitive impairment: a review. Neuroradiology 52:711-721 